\title{
Imaging technology acts as a 'virtual skin biopsy' in SSc
}

"Assessment of skin fibrosis is a crucial prognostic factor in SSc," says Francesco Del Galdo, from the University of Leeds, UK, "but at present there is no objective validated biomarker to assess this." The quest for such a biomarker is ongoing and, according to a paper from Del Galdo and colleagues in Annals of the Rheumatic Diseases, might have been achieved in the form of optical coherence tomography (OCT).

The current gold standard for assessing skin thickness in systemic sclerosis (SSc) is the modified Rodnan skin score (mRSS), which requires a high level of skill to perform and is subject to interobserver variability. "Our scope was to identify an imaging biomarker that could go beyond the assessment of overall skin thickness and offer a measure of fibrotic activity by detecting and hopefully quantifying the peculiar architectural changes of the skin that so far can be appreciated only by histology," explains Del Galdo.

OCT uses a low-intensity infrared laser beam to produce high-contrast images with resolutions of $4-10 \mu \mathrm{m}$ and can view as deep as $2 \mathrm{~mm}$ into the skin; the authors say this enables them to reconstruct a 'virtual skin biopsy'. 458 OCT scans of hands and forearms of 21 patients with SSc
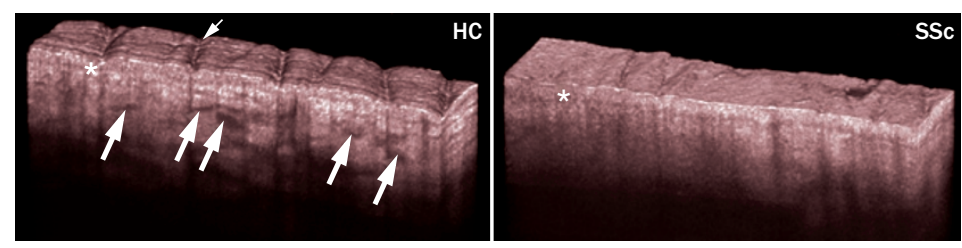

SS

and 22 healthy controls were performed and the optical density of the images were calculated and assessed. The OCT images were compared with skin histology findings from two patients with SSc $(\mathrm{mRSS}=3)$ and one healthy control.

In healthy skin, the epidermis (ED) and papillary dermis (PD) were easy to identify; the optical density of the PD was increased in comparison to that of the ED and the dermal-epidermal junction (DEJ) was clearly visible. Blood vessels were numerous. In the OCT images from patients with SSc, the ED and PD could not be easily distinguished between; the PD from patients with SSc did not have an increased optical density as seen in healthy skin and the DEJ was difficult to visualize. Blood vessels were less numerous than in healthy skin. The reduced optical density of the PD was more extreme in patients with higher mRSS scores, suggesting a progressive loss of DEJ visibility with more severe fibrosis.
"Our histology validation studies allowed us to determine that healthy skin shows a clear change in optical coherence at the DEJ and this is lost in severely affected SSc skin biopsies", says Del Galdo. "By OCT we were able to observe a consistent loss of visualization of the DEJ that could be objectively quantified by an automated software algorithm, correlated with skin thickness and showed excellent inter and intraobserver reliability".

The authors conclude that longitudinal studies assessing the sensitivity of OCT over time are needed to define the real potential of OCT to act as an imaging biomarker in SSc.

Jenny Buckland 